\title{
MOMENT COMPUTATION IN SHIFT INVARIANT SPACES
}

\author{
DAVID A. EUBANKS \\ Coker College \\ Department of Mathematics \\ Hartsville, SC 29550 USA \\ deubanks@pascal.coker.edu \\ PATRICK J. VAN FLEET ${ }^{1}$ and JIANZHONG WANG ${ }^{2}$ \\ Sam Houston State University \\ Department of Mathematics \\ Huntsville, TX 77341 USA \\ pvf@euler.shsu.edu,jwang@galois.shsu.edu
}

(Received December, 1996; Revised October, 1997)

\begin{abstract}
An algorithm is given for the computation of moments of $f \in S$, where $S$ is either a principal $h$-shift invariant space or $S$ is a finitely generated $h$ shift invariant space. An error estimate for the rate of convergence of our scheme is also presented. In so doing, we obtain a result for computing inner products in these spaces. As corollaries, we derive Marsden-type identities for principal $h$-shift invariant spaces and finitely generated $h$ shift invariant spaces. Applications to wavelet/multiwavelet spaces are presented.
\end{abstract}

Key words: (Principal) $h$-shift Invariant Space, Finitely Generated $h$ shift Invariant Space, Moment, Marsden's Identity, Wavelet, Multiwavelet.

AMS subject classifications: $41 \mathrm{~A} 25$.

\section{Introduction}

We consider the computation of the moment $m_{\beta}(f)$ of a function $f \in L^{2}(\mathbb{R})$. To this end, we project $f$ into either an $h$-principal invariant subspace or a finitely generated shift invariant subspace. The approximation order and other characteristics of such

\footnotetext{
${ }^{1}$ Research partially supported by National Science Grant DMS9503282 and the Texas Regional Institute for Environmental Studies.

${ }^{2}$ Research partially supported by the National Science Foundation Grant DMS9503282.
} 
spaces have been studied extensively in the fundamental paper [1] and again in [10, $11,15]$.

Our main result deals with the computation of inner products in shift invariant spaces. The advantage of utilizing these spaces is the fact that we can often construct stable bases whose elements are integer translates of a compactly supported function or a finite number of compactly supported functions. Thus the computation of the inner product is easily implemented on a computer. As corollaries to our main result, we obtain as a special case the ability to compute moments of functions $f_{0} \in V_{0}$. We then show how the process can be refined to obtain moments of the function $f_{h} \in V_{h}$. The idea is to construct a sequence of shift invariant spaces $V_{h}$ approximating $L^{2}(\mathbb{R})$ in hopes of eventually approximating the moment of $f \in L^{2}(\mathbb{R})$ by the moment of $f_{h} \in V_{h}$. In the case where the vector that generates the finitely generated shift invariant space $V_{0}$ is refinable, we use a result of Cohen, Daubechies, and Plonka [5] in order to obtain an estimate of the error $\left|m_{\beta}(f)-m_{\beta}\left(f_{h}\right)\right|$.

As a consequence of our main result, we characterize a Marsden's identity for finitely generated shift invariant spaces. Recall Marsden's identity gives the explicit representation of $x^{n}$ in terms of $B$-splines [12]. A multivariate analog for box splines is given in [3].

The outline of this paper is as follows: In Section 2, we give basic definitions and elementary results necessary to the sequel. An inner product theorem and related corollaries concerning moment computation and Marsden's identity are given in Section 3. In addition, we give an error estimate for the difference between the moment of $f \in L^{2}(\mathbb{R})$ and the moment of its orthogonal projection $f_{h}$ in a shift invariant subspace of $L^{2}(\mathbb{R})$. The final section contains moment recursion formulas for refinable functions and vectors as well as examples of illustrating our results.

\section{Notation, Definitions, and Basic Results}

In this section we introduce notation, definitions, and basic results used throughout the remainder of the paper. Let us begin by defining various types of shift invariant spaces.

Suppose $V_{h}$ is a linear space and $h>0$. Then $V_{h}$ is said to be an $h$-shift invariant space if

$$
f \in V_{h} \Rightarrow f(\cdot-h) \in V_{h} .
$$

$V_{h}$ is a principal h-shift invariant space if $V_{h}$ is an $h$-shift invariant space generated by a compactly supported function $\phi \in V_{h}$. That is,

$$
f \in V_{h} \Rightarrow f(x)=\sum_{k \in \mathbb{Z}} c_{k} \phi(x-h k) .
$$

When $h=1$, we will suppress the $h$ in the definitions above and refer to the spaces as shift invariant and principal shift invariant, respectively. As a matter of convention, we will denote an $h$-shift invariant space generated by $\phi$ by $V_{h}(\phi)$. We will also be interested in shift invariant spaces generated by several functions. That is, we define a finitely generated shift invariant space $V_{h}$ by insisting that

$$
f \in V_{h} \Rightarrow f(x)=\sum_{k \in \mathbb{Z}}\left(\mathbf{a}^{k}\right)^{T} \phi(x-h k),
$$


where now

$$
\phi(x)=\left(\begin{array}{c}
\phi_{1} \\
\vdots \\
\phi_{r}
\end{array}\right)(x),
$$

and the $\mathbf{a}^{k} \in \mathbb{R}^{r}$. Such a space generated by $\phi$ will be denoted by $V_{h}(\phi)$.

We readily observe the following properties:

(1) If $V$ is a shift invariant space, then $V_{h}=\{f(\cdot): f(h \cdot) \in V\}$ is an $h$-shift invariant space.

(2) If $V$ is a shift invariant space generated by $\phi$, then $V_{h}$ is an $h$-shift invariant space generated by $\phi(\dot{\bar{h}})$.

We will say that the $h$-shift invariant space $V_{h}$ is of degree $n$ and write $\operatorname{deg}\left(V_{h}\right)=n$ if $x^{k} \in V_{h}, k=0, \ldots, n$, but $x^{n+1} \notin V_{h}$. Since the degree of a polynomial is invariant under dilation, we observe that $\operatorname{deg}\left(V_{h}\right)=\operatorname{deg}(V)$.

If $\operatorname{deg}(V(\phi))=n$, we will call any identity of the form

$$
x^{\ell}=\sum_{k \in \mathbb{Z}} c_{k}^{\ell} \phi(x-k), \quad \ell=0, \ldots, n
$$

a Marsden's identity. The identity is easily generalized to the case where $V$ is generated by $\phi$.

Suppose $\phi$ generates the principal shift invariant space $V$. In this paper, we always assume that $\phi$ is integrable. Recall that if $\phi$ is compactly supported and integrable, then $\phi$ is also in $L^{2}$. Now we say a function $\phi^{*} \in V$ is the dual of $\phi$ if

$$
\left\langle\phi^{*}(\cdot-k), \phi(\cdot-j)\right\rangle=\delta_{k j}
$$

where $\langle f, g\rangle=\int_{\mathbb{R}} f(x) g(x) d x$ is the standard inner product and

$$
\delta_{k j}=\left\{\begin{array}{cc}
1 & \text { if } k=j \\
0 & \text { otherwise. }
\end{array}\right.
$$

Analogously, if vector $\phi$ generates the space $V$, then we will define its dual $\phi^{*} \in V$ as the vector of functions that satisfy

$$
\left\langle\phi_{\ell}^{*}(\cdot-k), \phi_{m}(\cdot-j)\right\rangle=\delta_{\ell m} \delta_{k j}
$$

We will say $\phi$ is stable if

$$
\sum_{k \in \mathbb{Z}}|\widehat{\phi}(\omega+2 k \pi)|^{2}>0 \quad \forall \omega \in \mathbb{R}
$$

and vector $\phi$ is stable if the $r \times r$ matrix $\widehat{\boldsymbol{\Phi}}:=\left(\widehat{\Phi}_{i j}(\omega)\right)_{i, j=1}^{r}$ is positive definitive where

$$
\widehat{\boldsymbol{\Phi}}_{i j}(\omega)=\sum_{k \in \mathbb{Z}} \widehat{\boldsymbol{\Phi}}_{i}(\omega+2 k \pi) \overline{\widehat{\boldsymbol{\Phi}}_{j}(\omega+2 k \pi)} .
$$

Recall that if $\phi$ is stable, then $\phi^{*}$ also generates a stable basis for $V$.

Suppose $\left\{\phi_{\ell}(\cdot-k)\right\}, k \in \mathbb{Z}, \ell=1, \ldots, r$ forms a basis for $V$. Then it also forms a basis for $V \cap L^{2}(\mathbb{R})$. For convenience, we will use $V$ to denote $V \cap L^{2}(\mathbb{R})$. We say that $V_{h}$ provides $L^{2}$-approximation order $m$ if, for each sufficiently smooth function 
$f \in L^{2}(\mathbb{R})$,

$$
\left\|f-\operatorname{Proj}_{V} f\right\|_{2} \leq C h^{m} \text {. }
$$

When $\phi$ is a compactly supported vector in $L^{2}$, then $V(\phi)$ provides $L^{2}$-approximation order $m$ if and only if $V(\phi)$ contains $\Pi_{m-1}$, the set of all polynomials of degree $\leq m-1[11]$.

Remark: If $\phi$ is refinable, i.e.,

$$
\phi(x)=\sum_{k=0}^{N} P_{k} \phi(2 x-k)
$$

where $P_{k}$ are $r \times r$ matrices, then $\Pi_{m-1} \subset V(\phi)$ is equivalent to the existence of solutions to two systems of equations involving the refinement mask of $\phi$ (see $[5,10$, 15]).

When $\phi$ is refinable in the sense of (1), we can obtain estimates on the accuracy of approximating moments of $f \in L^{2}(\mathbb{R})$ using projections of $f$ into $V_{h}(\phi)$.

Proposition 2.1: Suppose $\phi \in \mathbb{R}$ is a refinable vector and assume $\left\{\phi_{j}(\cdot-k)\right.$ : $k \in \mathbb{Z}, j=1, \ldots, r\}$ forms a linearly independent basis for the space $V(\phi)$ with $\operatorname{deg}(V(\phi))=n-1$. Further suppose that $f \in L^{2}(\mathbb{R})$ is compactly supported and sufficiently smooth. We have:

$$
\left|m_{\beta}(f)-m_{b}\left(\operatorname{Proj}_{V_{h}} f\right)\right| \leq C_{f} h^{n}
$$

where $\beta=0, \ldots, n-1$ and $C_{f}$ is a constant that depends on the support width of $f$.

Proof: The result of [5] guarantees that $V$ provides approximation order $n$. If $S$ denotes the support of $f$, we have

$$
\begin{gathered}
\left|m_{\beta}(f)-m_{\beta}\left(\operatorname{Prog}_{V_{m}} f\right)\right|=\left|\left\langle x^{\beta} \chi_{S}(x),\left(f-\operatorname{Proj}_{V_{m}} f\right)\right\rangle\right| \\
\leq\left\|x^{\beta} \chi_{S}(x)\right\| \cdot\left\|f-\operatorname{Proj}_{V_{m}} f\right\| \leq C_{f} h^{m} .
\end{gathered}
$$

\section{Main Results}

The main goal of this paper is to provide an algorithm for computing moments in principal (or finitely generated) shift invariant spaces. Once the algorithm is in place, we will use it to attempt to approximate the moment $m_{\beta}(f)$ of $f \in L^{2}(\mathbb{R})$. In order to obtain formula for computing moments of $f_{0}=\operatorname{Proj}_{V} f$ and subsequent moments in refined spaces, we establish the following result.

Theorem 3.1: Suppose $\phi$ is compactly supported, stable and that $\phi^{*}$ is its dual. Denote by $V(\phi)$ the space generated by $\phi$. Assume $f \in V(\phi)$ satisfies the decay condition

and $g \in V(\phi)$ satisfies the growth condition

$$
|f(x)| \leq \frac{C}{1+|x|^{\alpha}}, \quad \alpha>1,
$$

$$
|g(x)| \leq C\left(1+|x|^{\beta}\right), \quad \alpha-\beta>1
$$

where $C$ is an absolute constant. Let

$$
f(x)=\sum_{k \in \mathbb{Z}}\left(a^{k}\right)^{T} \phi(x-k), \quad g(x)=\sum_{k \in \mathbb{Z}}\left(b^{k}\right)^{T} \boldsymbol{\phi}^{*}(x-k) .
$$




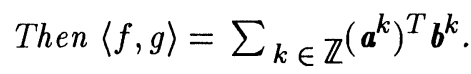

Proof: Without loss of generality, assume that $\operatorname{supp}(\phi)=[0, L]$. (By the support of a vector $\phi \in \mathbb{R}^{r}$, we mean $\operatorname{supp}(\phi)=\bigcup_{k=0}^{r} \operatorname{supp}\left(\phi_{k}\right)$.) First note that since $\phi$ is compactly supported, $\phi^{*}$ is of exponential decay. That is, for $k=1, \ldots, r$ :

$$
\left|\phi_{k}^{*}(x)\right| \leq C_{1} e^{-\gamma|x|}
$$

for some $\gamma>0$.

We shall now estimate the decay rate of $a_{i}^{k}$ and $b_{i}^{k}, k \in \mathbf{Z}$, and $i=1, \ldots, r$. First we show that for sufficiently large $k$ and constant $C_{2}>0$, we have

$$
\left|a_{i}^{k}\right| \leq C_{2}|k|^{-\alpha} \text {. }
$$

Since $\phi$ is compactly supported and stable, the set $\left\{\phi^{*}(\cdot-k)\right\}_{k \in \mathbb{Z}}$ forms an unconditional basis for $V(\phi)$. Therefore,

$$
a_{i}^{k}=\int_{\mathbb{R}} f(x) \phi_{i}^{*}(x-k) .
$$

Then for sufficiently large $k$, we have

$$
\begin{gathered}
\left|a_{i}^{k}\right|=\left|\int_{\mathbb{R}} f(x+k) \phi_{i}^{*}(x) d x\right| \\
\leq C_{1}\left(\int_{|x| \leq \frac{\alpha}{\gamma} \ln |k|} \frac{d x}{1+|x+k|^{\alpha}}+\int_{|x|>\frac{\alpha}{\gamma} \ln |k|} e^{-\gamma x} d x\right) \leq C_{2}|k|^{-\alpha} .
\end{gathered}
$$

Using an analogous argument, we have for constant $C_{3}>0$

$$
\left|b_{i}^{k}\right| \leq\left\{\begin{array}{cc}
C_{3}\left(1+|k+L|^{\beta}\right) & k \geq 0 \\
C_{3}\left(1+|k|^{\beta}\right) & k<0
\end{array}\right.
$$

Now for $M \in \mathbb{Z}$ and $M>0$ define

$$
f_{M}(x)=\sum_{k>M}\left(\mathbf{a}^{k}\right)^{T} \phi(x-k) \text { and } g_{M}(x)=\sum_{\ell>M}\left(\mathbf{b}^{\ell}\right)^{T} \boldsymbol{\phi}^{*}(x-\ell) .
$$

In an analogous manner, define $f_{-M}$ and $g_{-M}$. Then

Then

$$
\begin{gathered}
f(x)=\sum_{k=-M}^{M}\left(\mathbf{a}^{k}\right)^{T} \phi(x-k)+f_{-M}(x)+f_{M}(x) \\
g(x)=\sum_{\ell=-M}^{M}\left(\mathbf{b}^{\ell}\right)^{T} \boldsymbol{\phi}^{*}(x-\ell)+g_{-M}(x)+g_{M}(x) .
\end{gathered}
$$

$$
\int_{\mathbb{R}} f(x) g(x) d x=\int_{\mathbb{R}}\left(\sum_{k=-M}^{M}\left(\mathbf{a}^{k}\right)^{T} \boldsymbol{\phi}(x-k)\right)\left(\sum_{\ell=-M}^{M}\left(\mathbf{b}^{\ell}\right)^{T} \boldsymbol{\phi}^{*}(x-\ell)\right) d x
$$




$$
\begin{aligned}
& +\int_{\mathbb{R}}\left(\sum_{k=-M}^{M}\left(\mathbf{a}^{k}\right)^{T} \boldsymbol{\phi}(x-k)\right)\left(g_{-M}(x)+g_{M}(x)\right) d x \\
& +\int_{\mathbb{R}}\left(\sum_{\ell=-M}^{M}\left(\mathbf{b}^{\ell}\right)^{T}(x-\ell)\right)\left(f_{-M}(x)+f_{M}(x)\right) d x \\
& +\int_{\mathbb{R}}\left(f_{-M}(x)+f_{M}(x)\right)\left(g_{-M}(x)+g_{M}(x)\right) d x \\
& =\sum_{k=-M}^{M}\left(\mathbf{a}^{k}\right)^{T} \mathbf{b}+\int_{\mathbb{R}}\left(f_{-M}(x)+f_{M}(x)\right)\left(g_{-M}(x)+g_{M}(x)\right) d x .
\end{aligned}
$$

Using (7), (8) and the fact that $\alpha-\beta>1$ we see that the second term in the above sum tends to 0 as $M \rightarrow \infty$ so that we obtain the desired result.

We obtain the following moment formulas as immediate corollaries of Theorem 3.1 .

Corollary 3.2: Let $V(\phi)$ be generated by a compactly supported and stable $\phi$ and let $\phi^{*}$ be the dual of $\phi$. Further assume that $\operatorname{deg}(V(\phi))=n$,

$$
x^{\beta}=\sum_{k \in \mathbb{Z}}\left(c^{k, \beta}\right)^{T} \phi^{*}(x-k), \quad \beta=0, \ldots, n,
$$

and that $f \in V(\phi)$ satisfies the decay condition

$$
|f(x)| \leq \frac{C}{1+|x|^{n+\alpha}}
$$

where $\alpha-\beta>1$. Then

$$
m_{\beta}(f)=\sum_{k \in \mathbb{R}}\left(a^{k}\right)^{T} c^{k, \beta} .
$$

Corollary 3.2 illustrates how we may compute the moments of order $\beta$ or less of $f \in V(\phi)$. Note that in order to implement (11), we must have the coefficient vector $\mathrm{c}^{k, \beta}$ for $x^{\beta}$. We will discuss a procedure for obtaining $\mathrm{c}^{k, \beta}$ later in this section.

The following corollary describes how we can refine our procedure and obtain moments $m_{\beta}(f)$ where $f \in V_{h}(\phi)$.

Corollary 3.3: Let $f \in V_{h}(\phi)$ and have the representation

$$
f(x)=\sum_{k \in \mathbb{Z}}\left(a^{k}\right)^{T} \phi\left(\frac{x}{h}-k\right),
$$

with $h>0$. Further assume that $\operatorname{deg}\left(V_{h}(\phi)\right)=n$ and that $f$ satisfies the growth condition (1) from Corollary 3.2. Let $x^{\beta}$ be as in (9). Then

$$
m_{\beta}(f)=h^{\beta+1} \sum_{k \in \mathbb{Z}}\left(\boldsymbol{a}^{k}\right)^{T} \boldsymbol{c}^{k, \beta} .
$$

We continue by deriving explicit representations for $\mathbf{c}^{k, \beta}$. Assume that $\operatorname{deg}(V(\phi))=n$. Then for $\beta=0, \ldots, n, j=1, \ldots, r$, and $k \in \mathbf{Z}$ we have: 


$$
\begin{gathered}
c_{j}^{k, \beta}=\int_{\mathbb{R}} \phi_{j}(x-k) x^{\beta} d x=\int_{\mathbb{R}} \phi_{j}(x)(x+k)^{\beta} d x \\
=\sum_{\ell-0}^{\beta}\left(\begin{array}{c}
\beta \\
\ell
\end{array}\right) k^{\beta-\ell} \int_{\mathbb{R}} x^{\beta} \phi_{j}(x) d x \\
=\sum_{\ell=0}^{\beta}\left(\begin{array}{l}
\beta \\
\ell
\end{array}\right) k^{\beta-\ell_{m_{\beta}}\left(\phi_{j}\right) .}
\end{gathered}
$$

Thus to compute the $\mathbf{c}^{k, \beta}$, we need only the moments of order less than $\beta$ of the components of $\phi$. We shall see in the final section of the paper that in the case of the paper that in the case where $\phi$ is refinable then this task can be performed recursively. Once we have these moments, we can see Corollary 3.2 or Corollary 3.3 to compute moments of functions in (finitely generated) principal shift invariant subspaces of $L^{2}(\mathbb{R})$. In addition Proposition 2.1 provides a means to estimate moments from these spaces should we intend to use them to approximate moments of functions in $L^{2}(\mathbb{R})$.

We conclude this section by noting that in light of (9) and (13) we have the means for establishing a Marsden's identity in any finitely generated shift invariant space of degree $n$. Of course for computational purposes, we must also obtain explicit formula for the moments $m_{\beta}\left(\phi_{j}\right), \beta=0, \ldots, n$ and $j=1, \ldots, r$. Proposition 4.1 illustrates how we can obtain these values in the case where $r=1$ and the function $\phi$ solves (14). We give examples of particular vector functions in the next section.

\section{Refinable Functions and Vectors}

In the final section of the paper, we discuss various methods for computing the initial moments $m_{\beta}\left(\phi_{j}\right)$ as given in (13). One of the most popular ways to obtain classes of (finitely generated) principal shift invariant spaces is to use ideas from wavelet or multiwavelet theory (see $[2,6]$ for wavelets, $[8,9]$ for multiwavelets). The idea is to construct a nested ladder of principal shift invariant subspaces of $L^{2}(\mathbb{R})$. This ladder is constructed by finding a function $\phi$ (or a vector $\phi$ ) who along with its integer translates forms a Reisz basis for a space $V_{0}$. For $k \in \mathbf{Z}$, the space $V_{k}$ is formed using the translates $\left.\phi\left(2^{k} x-n\right), n \in \mathbf{Z}\right)$, of $\phi\left(2^{k} x\right)$. Other requirements are made of the nested ladder to ensure existence of a wavelet. The property we are particularly interested in is the refinement property (1).

Our first results of this section shows that if the generator $\phi$ is refinable, then we need only compute $\int \phi(x) d x$ and then this value to recursively generate all moments needed in (13).

Proposition 4.1: Assume that $\beta \geq 1, \beta \in \mathbf{Z}$, and that $\int \phi(x) d x \neq 0$. Furthermore suppose that there exists real numbers $p_{0}, \ldots, p_{N}$ so that $\mathbb{R}$

$$
\phi(x)=\frac{1}{2} \sum_{k=0}^{N} p_{k} \phi(2 x-k) .
$$

Then 


$$
m_{\beta}(\phi)=\frac{1}{2\left(2^{\beta}-1\right)} \sum_{\ell=0}^{\beta-1}\left(\begin{array}{l}
\beta \\
\ell
\end{array}\right)\left[\sum_{k=0}^{N} p_{k} k^{\beta-\ell}\right] m_{\ell}(\phi) \text {. }
$$

Proof: Multiply both sides of (14) by $x^{\beta}$ and integrate over $\mathbb{R}$. Upon simplification, we obtain

$$
m_{\beta}(\phi)=\frac{1}{\left(2^{\beta+1}-\sum_{k=0}^{N} p_{k}\right)} \sum_{\ell=0}^{\beta}\left(\begin{array}{l}
\beta \\
\ell
\end{array}\right)\left[\sum_{k=0}^{N} p_{k} k^{\beta-\ell}\right] m_{\ell}(\phi) .
$$
Take Fourier transforms of both sides of (14) and evaluate at 0 . Since $\int_{\mathbb{R}} \phi(x) d x \neq 0$,
we have

$$
\frac{1}{2} \sum_{k=0}^{N} p_{k}=1
$$

From which the result follows.

It is possible to generalize this result to the vector case. To this end, we introduce some new notation. Let $\boldsymbol{m}_{\beta}(\phi) \in \mathbb{R}^{r \times r}$ be the vector whose components are given by $\boldsymbol{m}_{\beta}(\phi)_{\ell}=m_{\beta}\left(\phi_{\ell}\right), \ell=1, \ldots, r$. In addition, we define the $r \times r$ matrix $P$ by

$$
P=\frac{1}{2} \sum_{k=0}^{N} P_{k}
$$

where the $P_{k}$ satisfy the refinement condition (1).

Proposition 4.2: Assume $\beta \geq 1, \beta \in \mathbf{Z}$ and that $\phi$ satisfies (1). Then $\boldsymbol{m}_{\beta}(\boldsymbol{\phi})$ can be obtained via recursion with $\boldsymbol{m}_{0}(\phi)$ as an initial starting point.

Proof: Multiply both sides of $(1)$ by $x^{\beta}$ and integrate over $\mathbb{R}$. Upon simplification we have:

$$
\boldsymbol{m}_{\beta}(\phi)=2^{-\beta-1} \sum_{j=0}^{\beta}\left(\begin{array}{l}
\beta \\
j
\end{array}\right)\left[\sum_{k=0}^{N} P_{k} k^{\beta-j}\right] \boldsymbol{m}_{j}(\phi) .
$$

Further simplification yields

$$
\left(I-2^{-\beta} P\right) \boldsymbol{m}_{\beta}(\phi)=\frac{1}{2} \sum_{j=0}^{\beta-1}\left(\begin{array}{l}
\beta \\
j
\end{array}\right)\left[\sum_{k=0}^{N} P_{k} k^{\beta-j}\right] \boldsymbol{m}_{j}(\phi) .
$$

It is shown in [13] that $\sigma_{P}$, the spectral radius of $P$ satisfies $\sigma_{P}=1$. Thus $\left(I-2^{-\beta} P\right)^{-1}$ exists.

The propositions above illustrate that we can use functions from wavelet theory and multiwavelet theory to approximate moments of functions in $L^{2}(\mathbb{R})$. Daubechies ([6]) has created a family of functions that can posses arbitrary regularity. Chui and Wang ([4]) have derived wavelets from cardinal $B$-splines. In term of multiwavelets, one could use Proposition 4.2 with the spline multiwavelets of Goodman and Lee ([9]) or the fractal multiwavelets given in ([7]).

We conclude the paper with two examples from the scaling functions listed in the previous paragraph. In both cases, we shall attempt to estimate moments of the Dirichlet density

$$
f(x ; a, b)=\left\{\begin{array}{cc}
x^{a}(1-x)^{b} / B(a, b), & x \in[0,1] \\
0 & \text { otherwise }
\end{array}\right.
$$


where $B(a, b)$ is the beta function and $a, b \in \mathbb{R}$ with $a, b>0$.

Example 4.3: We consider the family of Daubechies scaling functions $\phi^{2}, \ldots, \phi^{5}$ [6] (see Figure 1 below). $V_{0}^{\ell}$ is the closed linear span of the integer translates of $\phi^{\ell}$, $\ell=2, \ldots, 5$. Note that $V_{j}^{\ell} \subset V_{j+1}^{\ell}$ and that $\operatorname{deg}\left(V_{j}^{\ell}\right)=\ell-2$.
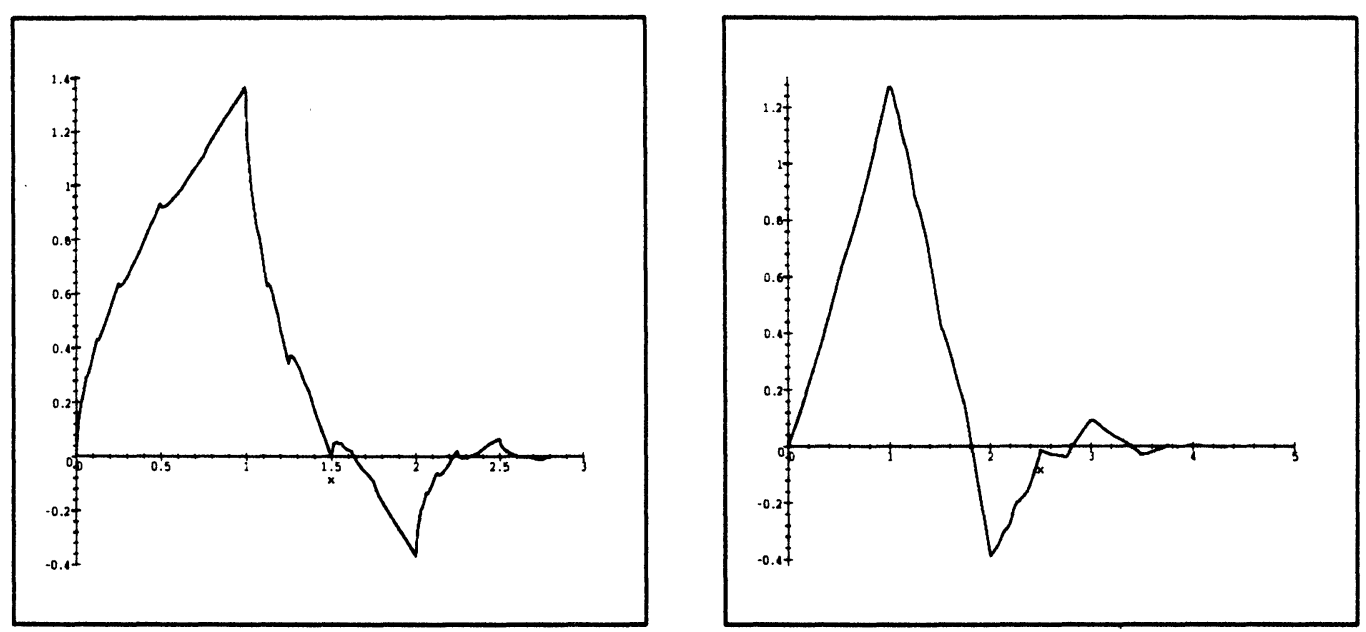

The functions $\phi^{2}$ (left) and $\phi^{3}$
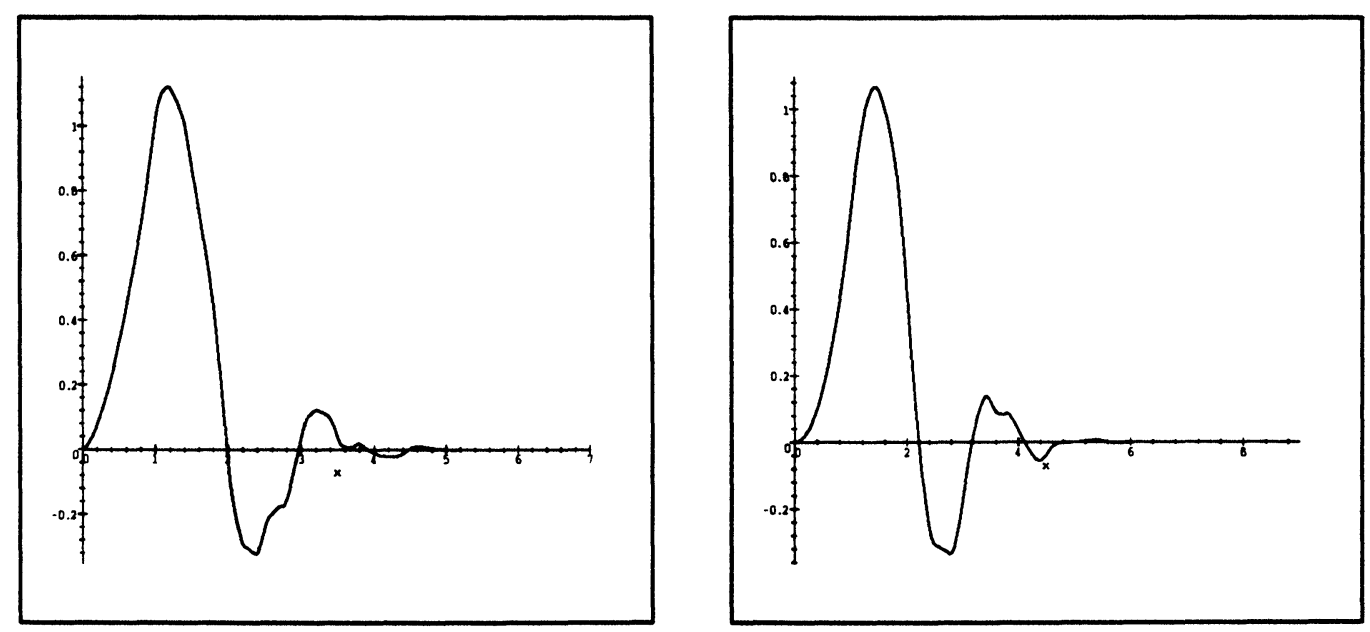

The functions $\phi^{4}(\mathrm{left})$ and $\phi^{5}$

Figure 1. Scaling functions for Example 4.3 
We will estimate the $\beta=1,2,3,4$ moments of $f\left(\cdot ; \frac{1}{2}, \frac{1}{2}\right)$ using each of $\phi_{2}, \ldots, \phi_{5}$. In order to do so, we must calculate the 0 order moment for each scaling function. We can then use the recursion formula in Proposition 4.1 to compute the higher order moments that are used to form the $c^{k, \beta}$. The next step is to obtain the $a^{k}$ s in Corollary 3.3. We provide our results for $h=2^{-j}$, where $j=6,8,10$.

We have provided three different methods for obtaining the $a^{k}$. In the first case, we simply sample $f$. In the second case, we approximate $f$ by a piecewise quadratic polynomial and then use the precomputed $c^{k, \beta}$ to form a Newton-Cotes type integration scheme. The third method for computing the $a^{k}$ uses the numerical integration from the prior method but uses a more sensitive approximation to $f$ at the breakpoints $x=0$ and $x=1$.

Our results are given in the tables below. The numbers in parentheses represent the error between the approximation and the exact value. Note that we have used $\phi^{2}, \phi^{3}$, and $\phi^{4}$ even in cases where Corollary 3.2 does not apply (that is, the order of the moment is larger than the degree of the space). The error in these cases is larger since $x^{\beta}$ is not a member of the spaces generated by the corresponding scaling functions. In addition, since we must approximate the $\left\{a^{k}\right\}$ in some fashion, the errors are dependent on the function $f$. Since $f$ is only $C^{0}$, it is nature that the $C^{0}$ function $\phi^{2}$ does an adequate job approximating the moments. Since the support of $\phi^{2}$ is less than that of any other scaling function we use, the expansions for $f_{h}$ consist of fewer terms. Thus, the computational cost of using $\phi^{2}$ is less than that incurred by the other scaling functions.

The actual moments of $f$ for this example are $m_{1}(f)=.5, m_{2}(f)=.3125$, $m_{3}(f)=.21875$, and $m_{4}(f)=.1640625$.

Daubechies' $\phi^{2}$ function

\begin{tabular}{|l|l|l|l|}
\hline$\beta$ & $h=2^{-6}$ & $h=2^{-8}$ & $h=2^{-10}$ \\
\hline 1 & $0.50230275(+4.61 e-03)$ & $0.50055824(+1.12 e-03)$ & $0.50013547(+2.71 e-04)$ \\
\hline 2 & $0.30729056(-1.67 e-02)$ & $0.31112133(-4.41 e-03)$ & $0.31214812(-1.13 e-03)$ \\
\hline 3 & $0.21142351(-3.35 e-02)$ & $0.21684828(-8.69 \mathrm{e}-03)$ & $0.21826775(-2.20 e-03)$ \\
\hline 4 & $0.15614446(-4.83 e-02)$ & $0.16200804(-1.25 e-02)$ & $0.16354169(-3.17 e-03)$ \\
\hline
\end{tabular}

Daubechies' $\phi^{3}$ function

\begin{tabular}{|l|l|l|l|}
\hline$\beta$ & $h=2^{-6}$ & $h=2^{-8}$ & $h=2^{-10}$ \\
\hline 1 & $0.48950970(-2.10 e-02)$ & $0.49736740(-5.27 e-03)$ & $0.49933799(-1.32 e-03)$ \\
\hline 2 & $0.29483178(-5.65 e-02)$ & $0.30795063(-1.46 e-02)$ & $0.31135186(-3.67 e-03)$ \\
\hline 3 & $0.20000283(-8.57 e-02)$ & $0.21389255(-2.22 \mathrm{e}-02)$ & $0.21752234(-5.61 e-03)$ \\
\hline 4 & $0.14570952(-1.12 e-01)$ & $0.15926406(-2.92 e-02)$ & $0.16284690(-7.41 e-03)$ \\
\hline
\end{tabular}




\section{Daubechies' $\phi^{4}$ function}

\begin{tabular}{|l|l|l|l|}
\hline$\beta$ & $h=2^{-6}$ & $h=2^{-8}$ & $h=2^{-10}$ \\
\hline 1 & $0.47679350(-4.64 e-02)$ & $0.49419456(-1.16 e-02)$ & $0.49854499(-2.91 e-03)$ \\
\hline 2 & $0.28276572(-9.51 e-02)$ & $0.30481785(-2.46 e-02)$ & $0.31056136(-6.20 e-03)$ \\
\hline 3 & $0.18911003(-1.35 e-01)$ & $0.21098329(-3.55 \mathrm{e}-02)$ & $0.21678298(-8.99 e-03)$ \\
\hline 4 & $0.13589634(-1.72 e-01)$ & $0.15657257(-4.57 e-02)$ & $0.16215836(-1.16 e-02)$ \\
\hline
\end{tabular}

Daubechies' $\phi^{5}$ function

\begin{tabular}{|l|l|l|l|}
\hline$\beta$ & $h=2^{-6}$ & $h=2^{-8}$ & $h=2^{-10}$ \\
\hline 1 & $0.46408879(-7.18 e-02)$ & $0.49102384(-1.80 e-02)$ & $0.49775250(-4.49 e-03)$ \\
\hline 2 & $0.27102926(-1.33 e-01)$ & $0.30170717(-3.45 e-02)$ & $0.30977261(-8.73 e-03)$ \\
\hline 3 & $0.17867438(-1.83 e-01)$ & $0.20810555(-4.87 \mathrm{e}-02)$ & $0.21604598(-1.24 e-02)$ \\
\hline 4 & $0.12662787(-2.28 e-01)$ & $0.15391947(-6.18 e-02)$ & $0.16147259(-1.58 e-02)$ \\
\hline
\end{tabular}

Table 1: $a^{k}$ sampled from $f$

Daubechies' $\phi^{2}$ function

\begin{tabular}{|l|l|l|l|}
\hline$\beta$ & $h=2^{-6}$ & $h=2^{-8}$ & $h=2^{-10}$ \\
\hline 1 & $0.51540693(+3.08 e-02)$ & $0.50388603(+7.77 e-03)$ & $0.50097426(+1.95 e-03)$ \\
\hline 2 & $0.32038575(+2.52 e-02)$ & $0.31444970(+6.24 e-03)$ & $0.31298696(+1.56 e-03)$ \\
\hline 3 & $0.22358158(+2.21 e-02)$ & $0.21995940(+5.53 \mathrm{e}-03)$ & $0.21905338(+1.39 e-03)$ \\
\hline 4 & $0.16737420(+2.02 e-02)$ & $0.16490249(+5.12 e-03)$ & $0.16427415(+1.29 e-03)$ \\
\hline
\end{tabular}

Daubechies' $\phi^{3}$ function

\begin{tabular}{|l|l|l|l|}
\hline$\beta$ & $h=2^{-6}$ & $h=2^{-8}$ & $h=2^{-10}$ \\
\hline 1 & $0.51796103(+3.59 e-02)$ & $0.50386543(+7.73 e-03)$ & $0.50097173(+1.94 e-03)$ \\
\hline 2 & $0.32299014(+3.36 e-02)$ & $0.31442920(+6.17 e-03)$ & $0.31298446(+1.55 e-03)$ \\
\hline 3 & $0.22602114(+3.32 e-02)$ & $0.21993897(+5.44 \mathrm{e}-03)$ & $0.21905087(+1.38 e-03)$ \\
\hline 4 & $0.16964373(+3.40 e-02)$ & $0.16488212(+5.00 e-03)$ & $0.16427162(+1.27 e-03)$ \\
\hline
\end{tabular}




\section{Daubechies' $\phi^{4}$ function}

\begin{tabular}{|l|l|l|l|}
\hline$\beta$ & $h=2^{-6}$ & $h=2^{-8}$ & $h=2^{-10}$ \\
\hline 1 & $0.51797521(+3.60 e-02)$ & $0.50385567(+7.71 e-03)$ & $0.50097056(+1.94 e-03)$ \\
\hline 2 & $0.32301051(+3.36 e-02)$ & $0.31441960(+6.14 e-03)$ & $0.31298329(+1.55 e-03)$ \\
\hline 3 & $0.22606401(+3.34 e-02)$ & $0.21992972(+5.39 \mathrm{e}-03)$ & $0.21904971(+1.37 e-03)$ \\
\hline 4 & $0.16970560(+3.44 e-02)$ & $0.16487322(+4.94 e-03)$ & $0.16427047(+1.27 e-03)$ \\
\hline
\end{tabular}

Daubechies' $\phi^{5}$ function

\begin{tabular}{|l|l|l|l|}
\hline$\beta$ & $h=2^{-6}$ & $h=2^{-8}$ & $h=2^{-10}$ \\
\hline 1 & $0.51794667(+3.59 e-02)$ & $0.50384989(+7.70 e-03)$ & $0.50096988(+1.94 e-03)$ \\
\hline 2 & $0.32298667(+3.36 e-02)$ & $0.31441390(+6.12 e-03)$ & $0.31298263(+1.54 e-03)$ \\
\hline 3 & $0.22608993(+3.36 e-02)$ & $0.21992478(+5.37 \mathrm{e}-03)$ & $0.21904904(+1.37 e-03)$ \\
\hline 4 & $0.16977257(+3.48 e-02)$ & $0.16486901(+4.92 e-03)$ & $0.16426981(+1.26 e-03)$ \\
\hline
\end{tabular}

Table 2: $a^{k}$ obtained by numerical integration

Daubechies' $\phi^{2}$ function

\begin{tabular}{|l|l|l|l|}
\hline$\beta$ & $h=2^{-6}$ & $h=2^{-8}$ & $h=2^{-10}$ \\
\hline 1 & $0.50992095(+1.98 e-02)$ & $0.50388040(+7.76 e-03)$ & $0.50097352(+1.95 e-03)$ \\
\hline 2 & $0.31482643(+7.44 e-03)$ & $0.31444359(+6.22 e-03)$ & $0.31298622(+1.56 e-03)$ \\
\hline 3 & $0.21857369(-8.06 e-04)$ & $0.21995342(+5.50 \mathrm{e}-03)$ & $0.21905261(+1.29 e-03)$ \\
\hline 4 & $0.16291963(-6.97 e-03)$ & $0.16489665(+5.08 e-03)$ & $0.16427338(+1.29 e-03)$ \\
\hline
\end{tabular}

Daubechies' $\phi^{3}$ function

\begin{tabular}{|l|l|l|l|}
\hline$\beta$ & $h=2^{-6}$ & $h=2^{-8}$ & $h=2^{-10}$ \\
\hline 1 & $0.51390530(+2.78 e-02)$ & $0.50356705(+7.13 e-03)$ & $0.50093456(+1.87 e-03)$ \\
\hline 2 & $0.31910721(+2.13 e-02)$ & $0.31413720(+5.24 e-03)$ & $0.31294749(+1.43 e-03)$ \\
\hline 3 & $0.22229573(+1.62 e-02)$ & $0.21964928(+4.11 \mathrm{e}-03)$ & $0.21901395(+1.21 e-03)$ \\
\hline 4 & $0.16602164(+1.19 e-02)$ & $0.16459481(+3.24 e-03)$ & $0.16423478(+1.05 e-03)$ \\
\hline
\end{tabular}




\section{Daubechies' $\phi^{4}$ function}

\begin{tabular}{|l|l|l|l|}
\hline$\beta$ & $h=2^{-6}$ & $h=2^{-8}$ & $h=2^{-10}$ \\
\hline 1 & $0.50995077(+1.99 e-02)$ & $0.50312468(+6.25 e-03)$ & $0.50087968(+1.76 e-03)$ \\
\hline 2 & $0.31583967(+1.07 e-02)$ & $0.31371336(+3.88 e-03)$ & $0.31289319(+1.26 e-03)$ \\
\hline 3 & $0.21913903(+1.78 e-03)$ & $0.21923114(+2.20 \mathrm{e}-03)$ & $0.21895985(+9.59 e-04)$ \\
\hline 4 & $0.16305911(-6.12 e-03)$ & $0.16418253(+7.32 e-04)$ & $0.16418086(+7.21 e-04)$ \\
\hline
\end{tabular}

Daubechies' $\phi^{5}$ function

\begin{tabular}{|l|l|l|l|}
\hline$\beta$ & $h=2^{-6}$ & $h=2^{-8}$ & $h=2^{-10}$ \\
\hline 1 & $0.50516894(+1.03 e-02)$ & $0.50357991(+5.16 e-03)$ & $0.50081227(+1.62 e-03)$ \\
\hline 2 & $0.31214977(-1.12 e-03)$ & $0.31320175(+2.25 e-03)$ & $0.31282678(+1.05 e-03)$ \\
\hline 3 & $0.21570730(-1.39 e-02)$ & $0.21872938(-9.43 \mathrm{e}-05)$ & $0.21889378(+6.57 e-04)$ \\
\hline 4 & $0.15993477(-2.51 e-02)$ & $0.16369097(-2.26 e-03)$ & $0.16411512(+3.21 e-04)$ \\
\hline
\end{tabular}

Table 3: $a^{k}$ obtained by adaptive numerical integration

We now consider an example illustrating our methods with finitely generated shift invariant spaces. To this end, we employ the scaling vector comprised of fractal interpolation functions given in [7]. We also note that in the case of these functions, a recursion formula for the moments exists (see [14]) and could be used in place of Proposition 4.2.

Example 4.4: We consider the closed linear space $V_{0}$ spanned by the fractal interpolation functions $\phi_{1}$ and $\phi_{2}$ as derived in [7]. We choose $\phi_{1}, \phi_{2}$ so that $\operatorname{deg}\left(V_{0}\right)=3$. We obtain the $V_{j}$ spaces by taking the closed linear span of the set $\left\{2^{-u / 2} \phi_{\ell}\left(2^{j} x-k\right), \ell=1,2\right\}_{k \in \mathbf{Z}}$. As in Example 4.3, we approximate moments of the beta distribution.

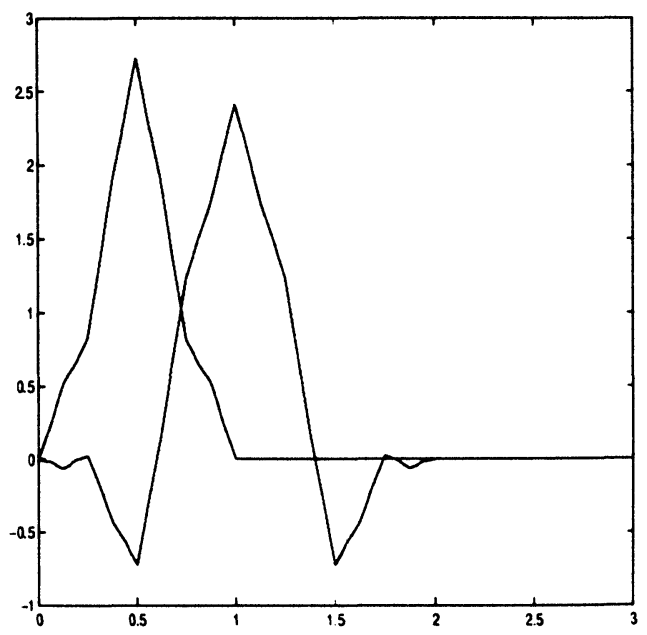

Figure 2: The fractal interpolation functions $\phi_{1}$ and $\phi_{2}$ 
Note that the accuracy is about the same as that of the Daubechies $\phi^{2}$ function. In the first table, the $a_{k}$ were function samples; in the second table, the $a_{k}$ were obtained using numerical integration; in the third table, the $a_{k}$ were obtained using adaptive numerical integration. The adaptive integration is not as effective here since one of the scaling functions has the same support as does $f$. The actual values for $m_{\beta}(f), \beta=1,2,3,4$ are given in the previous example.

$a_{k}$ from function samples

\begin{tabular}{|l|l|l|l|}
\hline$\beta$ & $h=2^{-6}$ & $h=2^{-8}$ & $h=2^{-10}$ \\
\hline 1 & $0.51082092(+2.16 e-02)$ & $0.50306346(+6.13 e-03)$ & $0.50107940(+2.16 e-03)$ \\
\hline 2 & $0.31564133(+1.01 e-02)$ & $0.31347012(+3.10 e-03)$ & $0.31293711(+1.40 e-03)$ \\
\hline 3 & $0.21909522(+1.58 e-03)$ & $0.21898027(+1.05 \mathrm{e}-03)$ & $0.21894533(+8.93 e-04)$ \\
\hline 4 & $0.16317880(-5.39 e-03)$ & $0.16395625(-6.48 e-04)$ & $0.16414017(+4.73 e-04)$ \\
\hline
\end{tabular}

$a_{k}$ obtained via numerical integration

\begin{tabular}{|l|l|l|l|}
\hline$\beta$ & $h=2^{-6}$ & $h=2^{-8}$ & $h=2^{-10}$ \\
\hline 1 & $0.51494589(+2.99 e-02)$ & $0.50382728(+7.65 e-03)$ & $0.50096687(+1.93 e-03)$ \\
\hline 2 & $0.31996907(+2.39 e-02)$ & $0.31439355(+6.06 e-03)$ & $0.31297975(+1.55 e-03)$ \\
\hline 3 & $0.22319232(+2.03 e-02)$ & $0.21990482(+5.28 \mathrm{e}-03)$ & $0.21904625(+1.35 e-03)$ \\
\hline 4 & $0.16700720(+1.79 e-02)$ & $0.16484914(+4.79 e-03)$ & $0.16426708(+1.25 e-03)$ \\
\hline
\end{tabular}

$a_{k}$ obtained via adaptive numerical integration

\begin{tabular}{|l|l|l|l|}
\hline$\beta$ & $h=2^{-6}$ & $h=2^{-8}$ & $h=2^{-10}$ \\
\hline 1 & $0.51444314(+2.89 e-02)$ & $0.50376013(+7.52 e-03)$ & $0.50095837(+1.92 e-03)$ \\
\hline 2 & $0.31946792(+2.23 e-02)$ & $0.31432651(+5.84 e-03)$ & $0.31297124(+1.51 e-03)$ \\
\hline 3 & $0.22269420(+1.80 e-02)$ & $0.21983768(+4.97 \mathrm{e}-03)$ & $0.21903774(+1.32 e-03)$ \\
\hline 4 & $0.16651210(+1.49 e-02)$ & $0.16478230(+4.39 e-03)$ & $0.16425857(+1.20 e-03)$ \\
\hline
\end{tabular}

Table 4: Moment computation from Example 4.4

\section{References}

[1] deBoor, C., DeVore, R. and Ron, A., Approximation from shift-invariant subspaces of $L_{2}\left(\mathbb{R}^{d}\right)$, Trans. Amer. Math. Soc. 341 (1994), 787-806.

[2] Chui, C.K., An Introduction to Wavelets, Academic Press, San Diego 1992.

[3] Chui, C.K. and Lai, M., A multivariate analog of Marsden's identity and a quasi-interpolation scheme, Const. Approx. 3 (1987), 111-122. 
[4] Chui, C.K. and Wang, J.Z., A general framework of compactly supported splines and wavelets, J. Approx. Th. 71 (1992), 263-304.

[5] Cohen, A., Daubechies, I. and Plonka, G., Regularity of refinable function vectors, preprint.

[6] Daubechies, I., Ten Lectures on Wavelets, SIAM CBMS Series No. 61, Philadelphia 1992.

[7] Donovan, G., Geronimo, J, Hardin, D. and Massopust, P., Construction of orthogonal wavelets using fractal interpolation functions, SIAM J. Math. Anal., to appear.

[8] Geronimo, J., Hardin, D. and Massopust, P., Fractal functions and wavelet expansions based on several scaling functions, J. Approx. Theory 78:3 (1994), 373-401.

[9] Goodman, T.N.T. and Lee, S.L., Wavelets of multiplicity r, Trans. Amer. Math. Soc. 342:1 (1994), 307-324.

[10] Heil, C., Strang, G. and Strela, V., Approximation by translates of refinable functions, Numer. Math., to appear.

[11] Jia, R.Q., Shift-invariant spaces on the real line, Proc. Amer. Math. Soc. 125:3 (1997), 785-793.

[12] Marsden, M.J., An identity for spline functions with applications to variationdiminishing spline approximation, J. Approx. Th. 3 (1970), 7-49.

[13] Massopust, P.R., Ruch, D.K. and Van Fleet, P.J., On the support properties of scaling vectors, Comp. and Appl. Harm. Anal. 3 (1996), 229-238.

[14] Massopust, P.R. and Van Fleet, P.J., On the moments of fractal functions and Dirichlet spline functions, preprint.

[15] Plonka, G., Approximation properties of multi-scaling functions: A Fourier approach, Constr. Approx., to appear. 


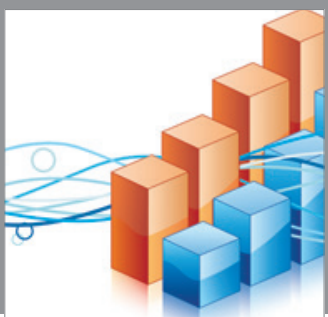

Advances in

Operations Research

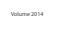

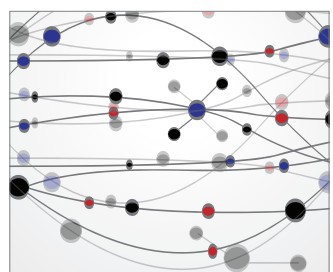

\section{The Scientific} World Journal
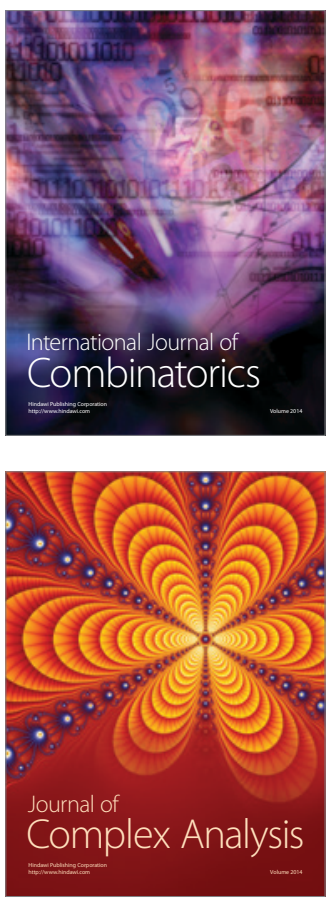

International Journal of

Mathematics and

Mathematical

Sciences
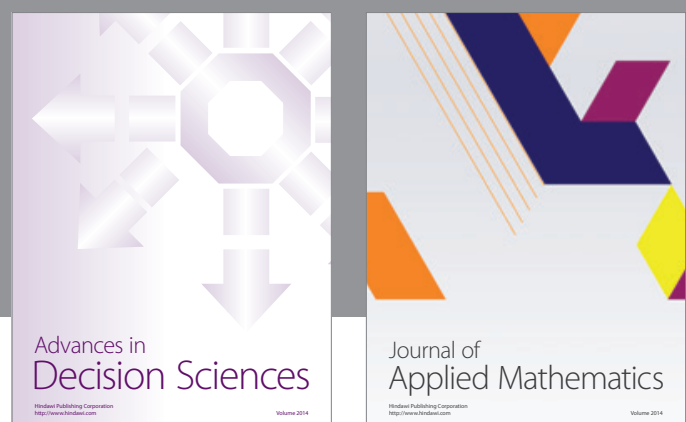

Journal of

Applied Mathematics
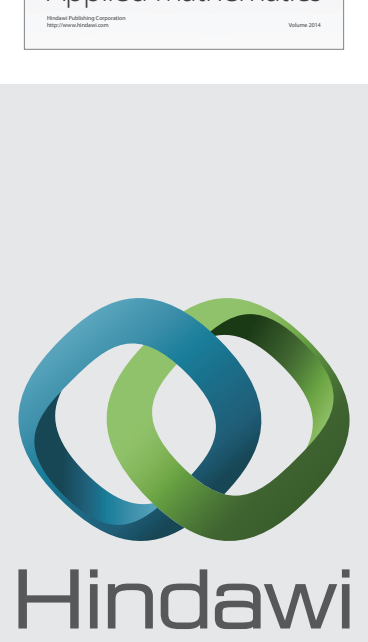

Submit your manuscripts at http://www.hindawi.com
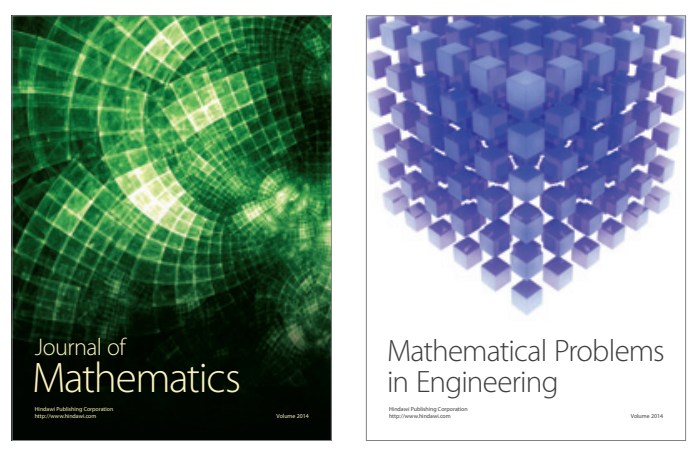

Mathematical Problems in Engineering
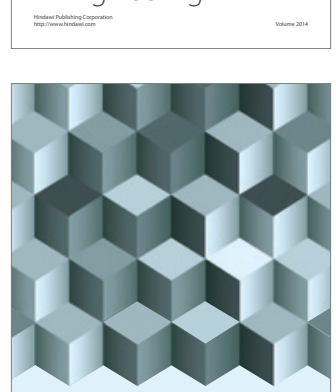

Journal of

Function Spaces
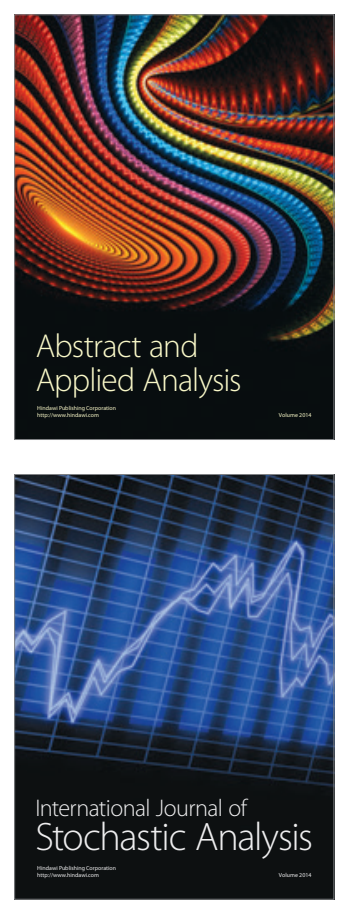

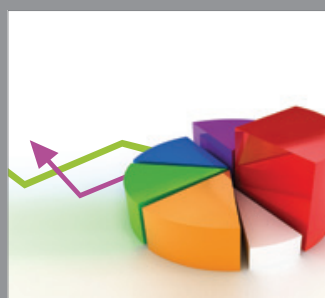

ournal of

Probability and Statistics

Promensencen
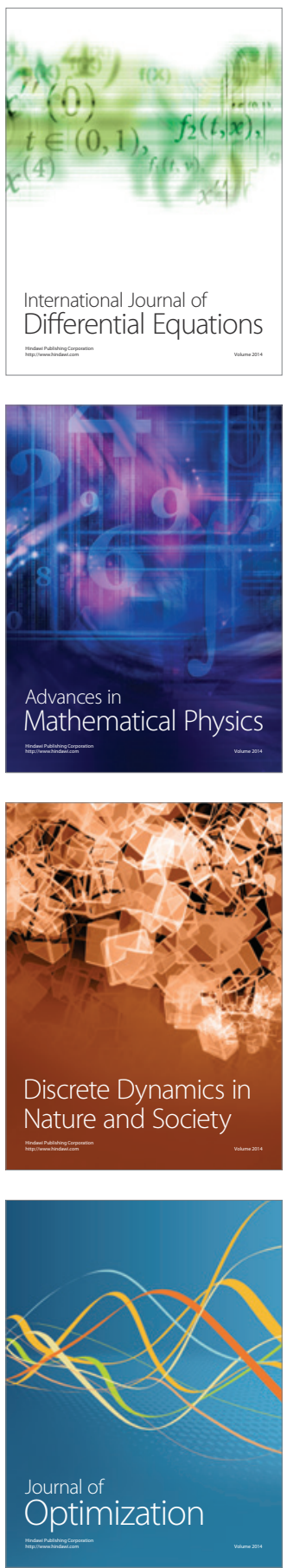